ARTICLE

https://doi.org/10.1038/s41467-019-12510-0

\title{
A universal ligand mediated method for large scale synthesis of transition metal single atom catalysts
}

\author{
Hongzhou Yang ${ }^{1,2,6}$, Lu Shang ${ }^{1,6}$, Qinghua Zhang ${ }^{3}$, Run Shi ${ }^{1}$, Geoffrey I.N. Waterhouse ${ }^{4}$, Lin Gu (1D ${ }^{3,5} \&$
}

Tierui Zhang (1) ${ }^{1,2 \star}$

There is interest in metal single atom catalysts due to their remarkable activity and stability. However, the synthesis of metal single atom catalysts remains somewhat ad hoc, with no universal strategy yet reported that allows their generic synthesis. Herein, we report a universal synthetic strategy that allows the synthesis of transition metal single atom catalysts containing $\mathrm{Cr}, \mathrm{Mn}, \mathrm{Fe}, \mathrm{Co}, \mathrm{Ni}, \mathrm{Cu}, \mathrm{Zn}, \mathrm{Ru}$, Pt or combinations thereof. Aberration-corrected high-angle annular dark-field scanning transmission electron microscopy and extended $\mathrm{X}$-ray absorption fine structure spectroscopy confirm that the transition metal atoms are uniformly dispersed over a carbon black support. The introduced synthetic method allows the production of carbon-supported metal single atom catalysts in large quantities ( $>1 \mathrm{~kg}$ scale) with high metal loadings. A Ni single atom catalyst exhibits outstanding activity for electrochemical reduction of carbon dioxide to carbon monoxide, achieving a $98.9 \%$ Faradaic efficiency at $-1.2 \mathrm{~V}$.

\footnotetext{
${ }^{1}$ Key Laboratory of Photochemical Conversion and Optoelectronic Materials Technical Institute of Physics and Chemistry, Chinese Academy of Sciences, 100190 Beijing, China. ${ }^{2}$ Center of Materials Science and Optoelectronics Engineering, University of Chinese Academy of Sciences, 100049 Beijing, China. ${ }^{3}$ Beijing National Laboratory for Condensed Matter Physics, Institute of Physics, Chinese Academy of Sciences, 100190 Beijing, China. ${ }^{4}$ School of Chemical Sciences, The University of Auckland, Auckland 1142, New Zealand. ${ }^{5}$ Songshan Lake Materials Laboratory, 523808 Dongguan, China. ${ }^{6}$ These authors contributed equally: Hongzhou Yang, Lu Shang. *email: tierui@mail.ipc.ac.cn
} 
H omogeneous and heterogeneous catalysts are widely used across chemical industry ${ }^{1-3}$. Homogeneous catalysts offer high efficiency and high selectivity due to their easily accessible active sites and maximal active metal utilization but suffer from low stability and poor recyclability. In contrast, heterogeneous catalysts offer excellent stability and good recyclability, but their active metal utilization is typically low (only surface atoms in supported metal nanoparticles actually participate in catalytic reactions) ${ }^{4,5}$. Single-atom catalysts (SACs) have recently emerged as a promising new class of catalytic materials, capturing the inherent advantages of both homogeneous and heterogeneous catalysts. SACs typically comprise individual metal atoms (M) immobilized on a support, with many SACs being stabilized through porphyrin-like $\mathrm{MN}_{4}$ surface coordination geometries $^{6}$. Accordingly, SACs have the dual advantage of nearly $100 \%$ atomic utilization (similar to homogeneous catalysts) as well as high stability and easy separation from reaction media (features of heterogeneous catalysts). Furthermore, when the size of active metal component is reduced from the nanometer lengths (i.e., nanoparticles or clusters) to the atomic level (i.e., SACs), new electronic states of the metal can appear thereby imparting unique performance ${ }^{7}$.

Following the pioneering discovery of SACs by Zhang et al. ${ }^{8}$, numerous experimental and theoretical studies have highlighted the outstanding catalytic potential of SACs for hydrogenation reactions ${ }^{9}$, carbon monoxide $(\mathrm{CO})$ oxidation ${ }^{10}$, methane conversion ${ }^{11}$, carbon dioxide $\left(\mathrm{CO}_{2}\right)$ reduction ${ }^{12-16}$, hydrogen evolution ${ }^{17}$, oxygen reduction ${ }^{18}$, dinitrogen $\left(\mathrm{N}_{2}\right)$ reduction ${ }^{19}$, and other chemical transformations ${ }^{1}$. Various synthetic strategies have been used to access SACs, including physical and chemical methods. Physical methods, such as mass-selected soft landing 20 and atomic layer deposition ${ }^{21}$, are not especially amenable for large-scale production of SACs since they require complex and expensive equipment. Chemical strategies involving the atomic dispersion of metals on a catalyst support, typically through the adsorption of metal precursors followed by reduction and stabilization, are therefore more practical ${ }^{22}$. Another common chemical approach toward SACs involves the carbonization of zeolitic imidazolate frameworks containing Co (ZIF-67) and Zn $(\mathrm{ZIF}-8)^{23,24}$. Although the feasibility of these two synthetic approaches has been confirmed in a number of studies ${ }^{25}$, the low yields and low metal loadings ( $1 \mathrm{wt} . \%)$ of these approaches limit their practical usefulness. For many practical applications, SACs with high metal loadings are demanded. Further, existing chemical strategies are not very versatile and cannot easily be adapted to synthesize SACs containing other transition metals. Some recent studies have attempted to develop more universal synthetic methods toward SACs. Duan et al. used holey graphene as substrate to synthesize $\mathrm{Fe}, \mathrm{Co}$, and $\mathrm{Ni}-\mathrm{SACs}^{26}$. Qu et al. reported that $\mathrm{Cu}, \mathrm{Co}$, or Ni-SACs could be fabricated on a carbon support containing abundant defect sites (derived by pyrolysis of ZIF-8) using a gas-migration method ${ }^{27}$. However, a single synthetic strategy that allows the large-scale synthesis of SACs containing almost any transition metal with high metal loadings has proven elusive. Such a synthetic strategy would expedite the utilization of SACs across the chemical sector. Recently, Beller and co-workers ${ }^{28}$ reported a novel protocol for preparing nanoscale cobalt-based catalysts via pyrolysis of organometallic amine complexes on activated carbon. The coordination of the metal cations by ligands during the pyrolysis step was found to reduce Co agglomeration leading to the formation of ultrafine supported Co nanoparticles. However, the high temperature $\left(800^{\circ} \mathrm{C}\right)$ used in that study was an obstacle to obtaining SACs. By lowering the pyrolysis temperature, SACs should be accessible since the kinetics and thermodynamic driving force for metal nanoparticle formation should be greatly reduced, motivating a detailed investigation.

Herein we report the successful synthesis of a library of $\mathrm{M}$ SACs $(\mathrm{M}=\mathrm{Ni}, \mathrm{Mn}, \mathrm{Fe}, \mathrm{Co}, \mathrm{Cr}, \mathrm{Cu}, \mathrm{Zn}, \mathrm{Ru}, \mathrm{Pt}$, and combinations thereof) by complexing metal cations with 1,10-phenanthroline, adsorbing the resulting metal complexes onto commercial carbon black (Ketjenblack EC-300J), followed by pyrolysis of the resulting surface-modified carbons at $600^{\circ} \mathrm{C}$ under an argon atmosphere (Fig. 1, see "Methods" section for full details). Aberrationcorrected high-angle annular dark-field scanning transmission electron microscopy (HAADF-STEM) and extended X-ray absorption fine structure (EXAFS) spectroscopy confirm that all M-SACs contain metals atomically dispersed in porphyrin-like $\mathrm{MN}_{4}$ sites over the carbon support (no cluster or nanoparticle formation is observed). X-ray absorption near edge spectroscopy (XANES) and X-ray photoelectron spectroscopy (XPS) reveal that all M-SACs contained $\mathrm{M}^{2+}$ ions. Further, compositional analysis by inductively coupled plasma optical emission spectroscopy (ICP-OES) establish that the synthesis method could produce MSACs with high metal loadings (easily up to 1.8 wt.\% for all metals, Supplementary Table 1). The performance of the different M-SACs are subsequently evaluated for electrochemical $\mathrm{CO}_{2}$ reduction, with the Ni-SAC containing $2.5 \mathrm{wt} \%$ demonstrating outstanding performance for $\mathrm{CO}_{2}$ reduction to $\mathrm{CO}(\sim 99 \%$ Faradaic efficiency at $-1.2 \mathrm{~V}$ ).

\section{Results}

Characterization of M-SACs. The structure of the M-SACs synthesized using the new "ligand-mediated" method $(\mathrm{M}=\mathrm{Ni}$, $\mathrm{Mn}, \mathrm{Fe}, \mathrm{Co}, \mathrm{Cr}, \mathrm{Cu}, \mathrm{Zn}, \mathrm{Ru}, \mathrm{Pt}$, and combinations thereof) were first probed by powder X-ray diffraction (XRD) and transmission electron microscopy (TEM). The XRD patterns of all M-SACs showed broad peaks centered around $24^{\circ}$ and $44^{\circ}$ (Fig. 2a), corresponding to the (002) and (100) planes of graphite. No metalrelated peaks were observed in the XRD patterns. Conversely, pyrolysis of the Ni-SAC precursor at $800{ }^{\circ} \mathrm{C}$ resulted in the formation of some Ni clusters (Supplementary Fig. 1), in agreement with Beller's results ${ }^{28}$. It can be concluded that the lower pyrolysis temperature of $600{ }^{\circ} \mathrm{C}$ used in the current work prevented metal nanocluster formation. TEM images for the M-SACs confirmed that no metal clusters or nanoparticles were present on the carbon black support (Fig. 2b and Supplementary Figs. 2-9). Energydispersive X-ray (EDX) element maps (Fig. 2c-g) for Ni-SAC confirmed a very homogeneous dispersion of $\mathrm{Ni}$ and $\mathrm{N}$ over the

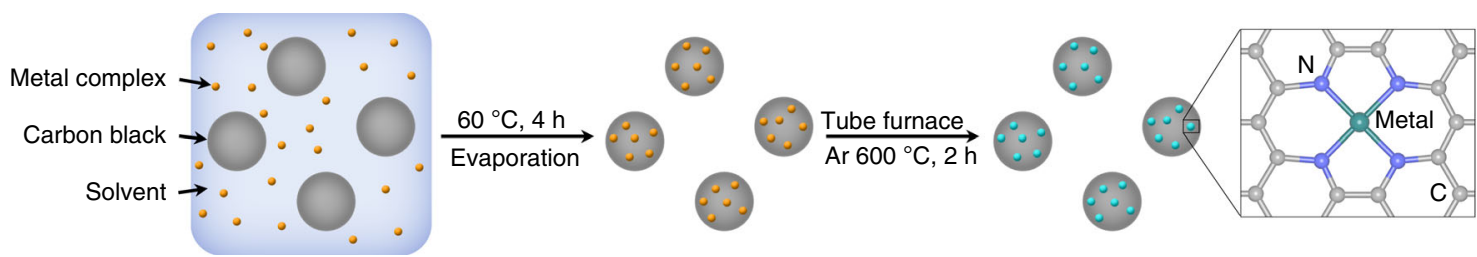

Fig. 1 The universal synthesis procedure. Metal single-atom catalysts (M-SACs) are prepared in two steps 

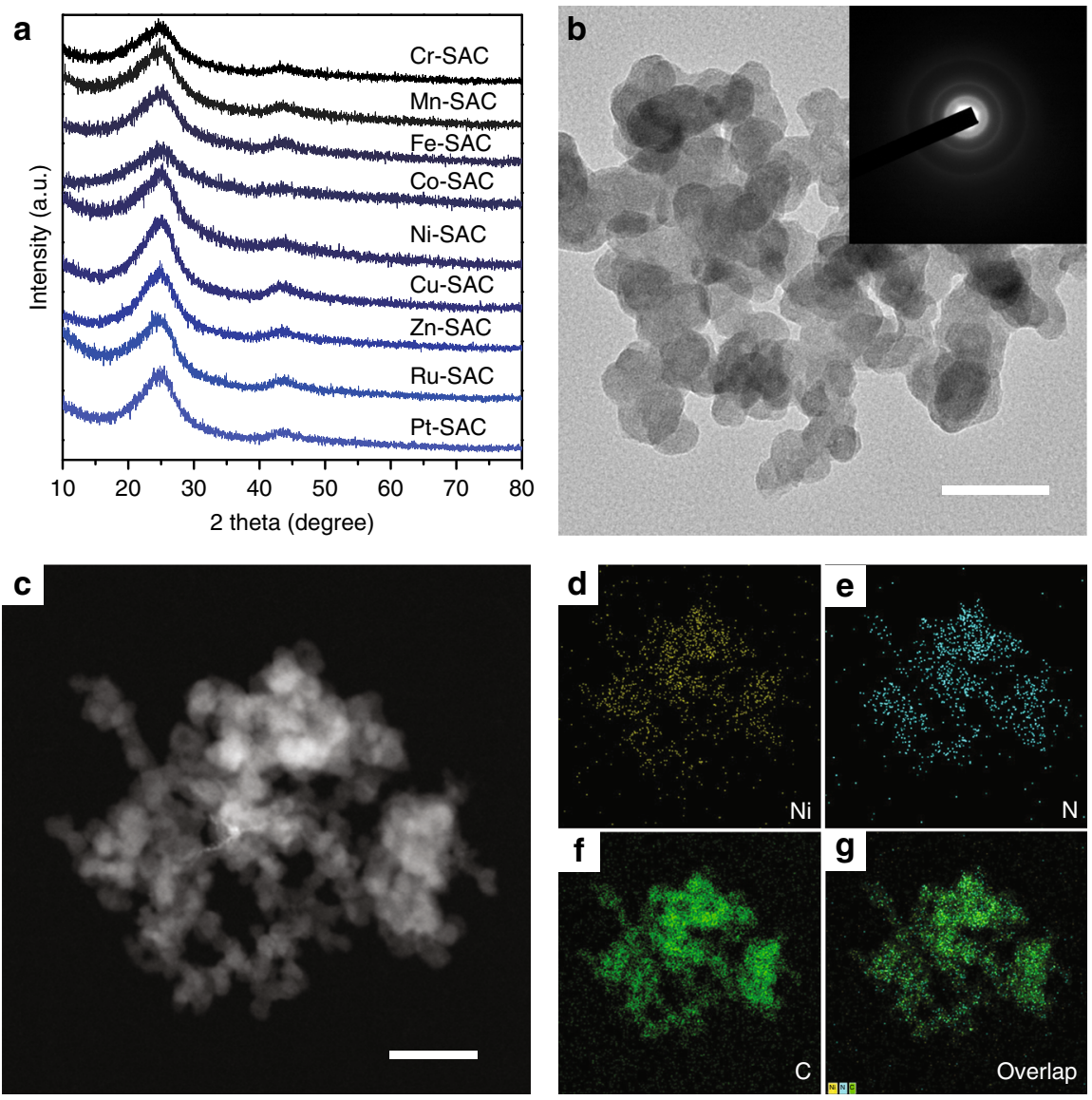

Fig. 2 Characterization of metal single-atom catalysts. a XRD patterns for different metal single-atom catalysts. b TEM image and SAED pattern (inner) for Ni-SAC-2.5, scale bar $100 \mathrm{~nm}$. c STEM image for Ni-SAC-2.5, scale bar $100 \mathrm{~nm}$. d-g EDX maps for Ni-SAC-2.5
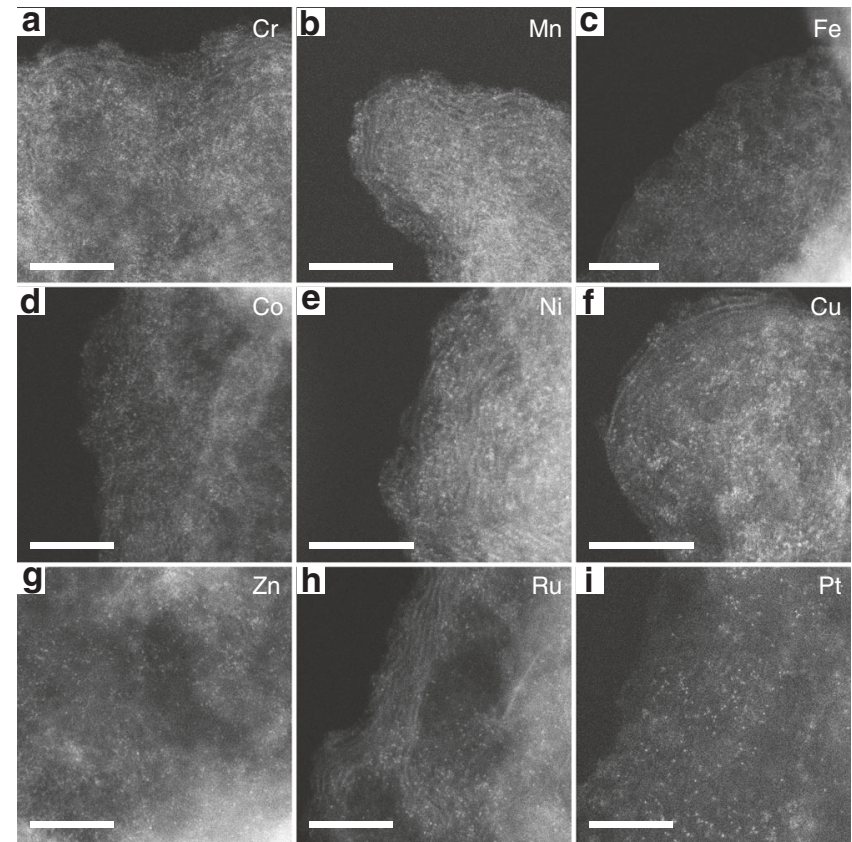

Fig. 3 Images for various metal single-atom catalysts. Aberration-corrected high-angle annular dark-field scanning transmission electron microscopy for a $\mathrm{Cr}-\mathrm{SAC}$, b Mn-SAC, c Fe-SAC, d Co-SAC, e Ni-SAC, f Cu-SAC, $\mathbf{g} \mathrm{Zn}-$ SAC, h Ru-SAC, and i Pt-SAC. Scale bar: $5 \mathrm{~nm}$ carbon support. Selected-area electron diffraction (Fig. 2b, inset) showed that the Ni-SAC possessed low crystallinity and no Ni nanoparticles (evidenced by an absence of any intense rings), consistent with the XRD results. The Raman spectrum for NiSAC (Supplementary Fig. 10) contained two peaks at 1359 and $1595 \mathrm{~cm}^{-1}$, which can readily be assigned to disordered $\mathrm{sp}^{3}$ carbon ( $D$ band) and graphitic $\mathrm{sp}^{2}$ carbon ( $\mathrm{G}$ band), respectively. The $I_{\mathrm{D}} / I_{\mathrm{G}}$ ratio for Ni-SAC $(0.87)$ was lower than that determined for pristine carbon black (1.01), indicating that the graphitic content of the carbon support increased after the pyrolysis step used to synthesize Ni-SAC.

HAADF-STEM was used to definitively confirm the atomic dispersion of metal atoms over the carbon support in the different M-SACs. As shown in the HAADF-STEM images of the SACs (Fig. 3a-g), the first row of transition metals (Cr, Mn, Fe, Co, Ni, $\mathrm{Cu}, \mathrm{Zn}$ ) appeared as high-density bright dots, indicating that these metals existed in the single-atom form on the carbon substrate. Similar results were found for the SACs containing the second-row $(\mathrm{Ru})$ and third-row $(\mathrm{Pt})$ transition metals (Fig. 3h, i, respectively), where $\mathrm{Ru}$ and $\mathrm{Pt}$ atoms were found to be homogeneously dispersed as single atoms on the carbon support. A further advantage of the synthetic strategy reported herein is that it allows the synthesis of multicomponent metal SACs, which are very difficult to obtain via conventional pyrolysis methods used to synthesize SACs. For example, bimetallic Fe/Co-SACs could be successfully synthesized. XRD (Supplementary Fig. 11) and HAADF-STEM (Supplementary Fig. 12) confirmed that Fe and Co atoms were atomically dispersed over the carbon support. $\mathrm{Ru} / \mathrm{Fe}-\mathrm{SACs}, \quad \mathrm{Ru} / \mathrm{Co}-\mathrm{SACs}$, and $\mathrm{Ru} / \mathrm{Ni}$-SACs were also 

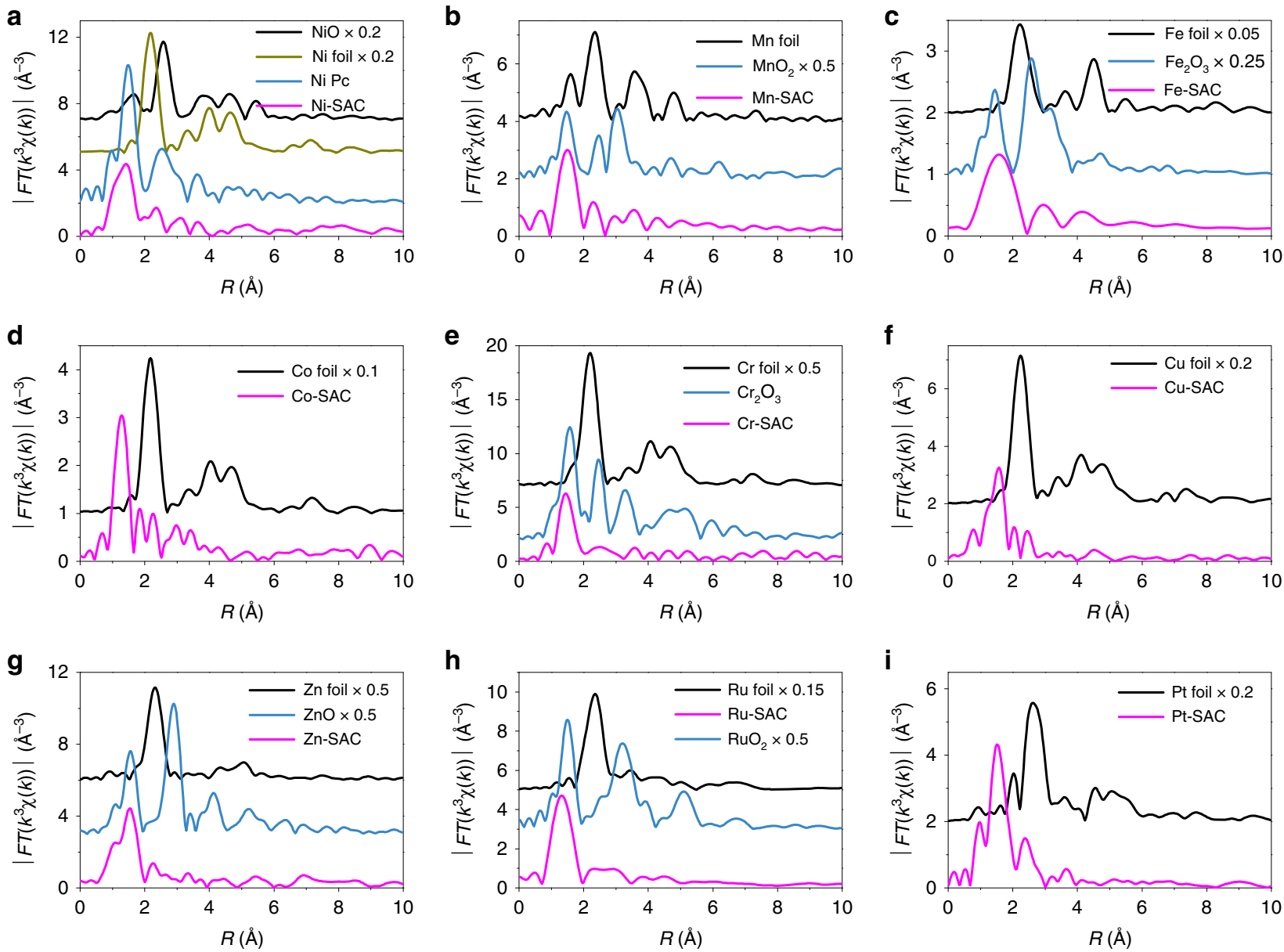

Fig. 4 Metal $K$-edge extended X-ray absorption fine structure ( $R$ space plots). a Ni-SAC, b Mn-SAC, c Fe-SAC, d Co-SAC, e Cr-SAC, f Cu-SAC, g Zn-SAC, h Ru-SAC, and $\mathbf{i}$ Pt-SAC. In all the M-SACs, the metal atoms existed as isolated $\mathrm{M}^{2+}$ centers in a $\mathrm{MN}_{4}$ coordination environment

synthesized. XRD patterns for these catalysts (Supplementary Fig. 13) contained two broad peaks corresponding to the carbon black support and no other features. These studies highlight the universality of the new synthesis method for the preparation of M-SACs.

EXAFS spectroscopy is very sensitive to the local environment of metal atoms. Accordingly, EXAFS was applied to confirm the presence of single metal atom sites in the various M-SACs. Figure 4 shows metal $K$-edge EXAFS data ( $R$ space plots) for the SACs along with data for relevant reference samples. For each $\mathrm{M}$ SAC, the $R$ space plots were quite distinct from that of the corresponding metal or metal oxide reference. Ni-SAC showed an intense peak at approximately $1.45 \AA$, corresponding to the first $\mathrm{Ni}-\mathrm{N}$ coordination shell, with the oscillation being very similar to that observed for nickel phthalocyanine (Ni Pc), indicating that $\mathrm{Ni}$ atoms in the Ni-SAC had a similar local environment with that of $\mathrm{Ni}$ atoms in Ni Pc. Fitting result determined that $\mathrm{Ni}$ in $\mathrm{Ni}$ SAC was coordinated fourfold by $\mathrm{N}$ atoms, as is also found in $\mathrm{Ni}$ Pc (Supplementary Fig. 14). The other M-SACs also showed only a single peak in $R$ space (at shorter lengths than the typical M-M distance in the corresponding metal foil), confirming that no metal-metal bonds existed in the M-SACs. These results were in good accord with the findings of the HAADF-STEM analyses. XANES spectra and $k^{3}$-weighted $K$-space spectra for the different M-SACs are shown in Supplementary Figs. 15-32. For all nine MSAC samples, the adsorption edge was higher than that of the corresponding metal foil, indicating that M-SACs contained metal atoms in cationic states (likely the $\mathrm{M}^{2+}$ state) ${ }^{29-31}$.
The chemical composition and elemental states of the M-SACs were investigated by XPS. The Ni $2 p$ XPS spectrum for Ni-SAC (Supplementary Fig. 33a) showed a $\mathrm{Ni} 2 \mathrm{p}_{3 / 2}$ peak at $855.6 \mathrm{eV}$, higher than that observed for $\mathrm{Ni}^{0}$ and slightly lower than that for $\mathrm{Ni}^{2+}$ in $\mathrm{NiO}$. The data provide strong evidence for the presence of $\mathrm{Ni}^{2+}$ in the $\mathrm{Ni}$-SACs, with the slightly lower binding energy compared to $\mathrm{NiO}$ due to coordination by $\mathrm{N}$ rather than $\mathrm{O}$. The $\mathrm{C}$ 1s spectrum (Supplementary Fig. 33b) was deconvoluted into two peaks, corresponding to $\mathrm{C}-\mathrm{C}$ or $\mathrm{C}=\mathrm{C}$ (neutral carbon and adventitious hydrocarbons) and $\mathrm{C}-\mathrm{N}$ species. The $\mathrm{N}$ 1s spectrum (Supplementary Fig. 33c) for the Ni-SAC could be deconvoluted into three peaks, corresponding to pyridinic-N, pyrrolic- $\mathrm{N}$, and graphitic- $\mathrm{N}$. The presence of $\mathrm{N}$, which originated from the 1,10 phenanthroline ligand, adds further weight to the proposal that the metal atoms in the M-SACs existed in $\mathrm{MN}_{4}$ coordination environments (as established from the EXAFS analyses). XPS data collected for the other M-SACs (Supplementary Figs. 34-41) were similar to that reported for the Ni-SAC, with the metal core-level spectra providing strong evidence for the presence of $\mathrm{M}^{2+}$ singleatom states.

Synthesizing SACs with high metal loadings and in large quantities has traditionally proved technically challenging and remains an obstacle to the widespread utilization of SACs. In traditional synthesis methods, SACs are obtained by hightemperature pyrolysis of metal organic frameworks (MOFs) or metal-impregnated graphitic carbon nitride $\left(\mathrm{g}-\mathrm{C}_{3} \mathrm{~N}_{4}\right)$. Such methods produce single-atom sites, though a large fraction of metal atoms in the precursor are typically transformed to metal 

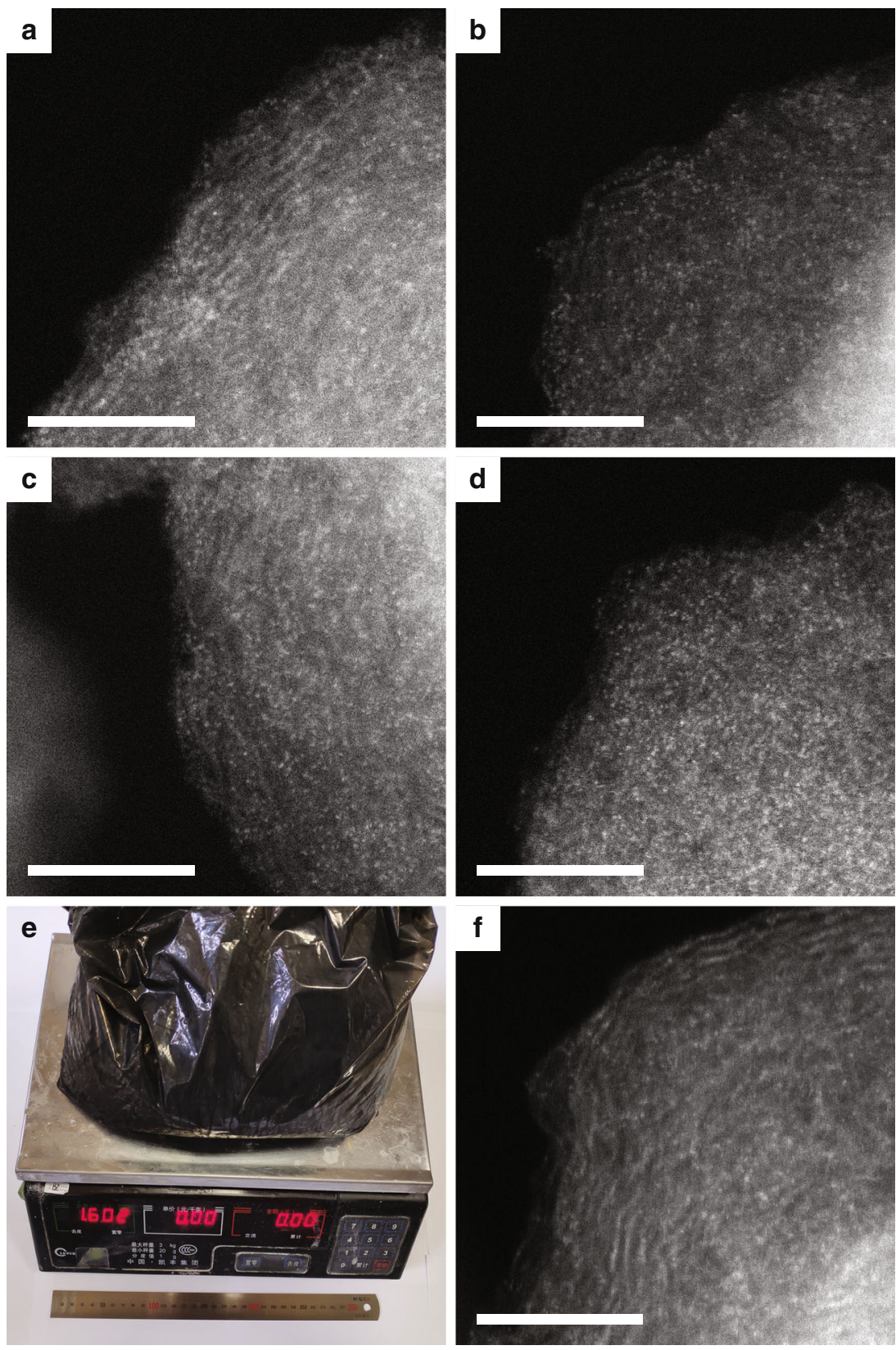

Fig. 5 Characterization and large-scale synthesis of Ni catalysts with different loadings. a HAADF-STEM image for Ni-SAC with 2.5 wt.\% Ni loading. b HAADF-STEM image for Ni-SAC with 3.4 wt.\% Ni loading. c HAADF-STEM image for Ni-SAC with 4.5 wt.\% Ni loading. d HAADF-STEM image for NiSAC with 5.3 wt.\% Ni loading. Scale bar: $5 \mathrm{~nm}$. e Photograph for Ni-SAC-2.5 synthesized in large scale. f HAADF-STEM image for Ni-SAC-2.5 synthesized in large scale. Scale bar: $5 \mathrm{~nm}$

clusters or nanoparticles. Here a low pyrolysis temperature of only $600^{\circ} \mathrm{C}$ was used to synthesize the M-SACs, which was possible since we used a commercial carbon as the support (thus higher temperatures were not needed to obtain a partially graphitic and conductive carbon support as is the case with MOFs or $\mathrm{g}_{-} \mathrm{C}_{3} \mathrm{~N}_{4}$ precursors). Accordingly, the method we developed was expected to be more amenable for the synthesis of M-SACs with high metal loadings. To test this hypothesis, we synthesized a series of Ni-SACs with different $\mathrm{Ni}$ loadings (denoted as Ni-SAC$x$, where $x$ is the actual $\mathrm{Ni}$ loading in wt.\%), with the actual $\mathrm{Ni}$ loadings determined by ICP-OES. Using our new "ligandmediated" synthetic strategy, Ni-SACs with Ni loadings of 2.5, $3.4,4.5$, and 5.3 wt. $\%$ were obtained, with the high loadings being superior to those of most other Ni-SACs reported to date (Supplementary Table 2). Supplementary Figs. 42-45 show TEM images for the Ni-SACs prepared at the different Ni loadings. For all Ni-SAC- $x$ samples, no metal nanoparticles were observed. The XRD patterns for each sample contained broad peaks at $24^{\circ}$ and $44^{\circ}$ (Supplementary Fig. 46) corresponding to the (002) and (100) planes of graphite. To confirm that Ni was atomically dispersed at each loading, HAADF-STEM and EXAFS analyses were performed. Figure 5a-d show HAADF-STEM images for the Ni-SAC- $x$ samples. For all samples, the atoms were atomically dispersed, with a few very small Ni clusters seen for the Ni-SAC5.3 sample. Supplementary Figs. 47-49 show Ni K-edge EXAFS $R$ space plots, Ni $K$-edge XANES, and Ni $K$-edge EXAFS $K$-space plots, respectively, for the Ni-SACs synthesized with different $\mathrm{Ni}$ loadings. The XANES data confirmed that all the Ni-SACs contained $\mathrm{Ni}^{2+}$. The $R$ space plots showed a distinct feature at $\sim 1.45 \AA$, corresponding to Ni-N first coordination shell. Results 

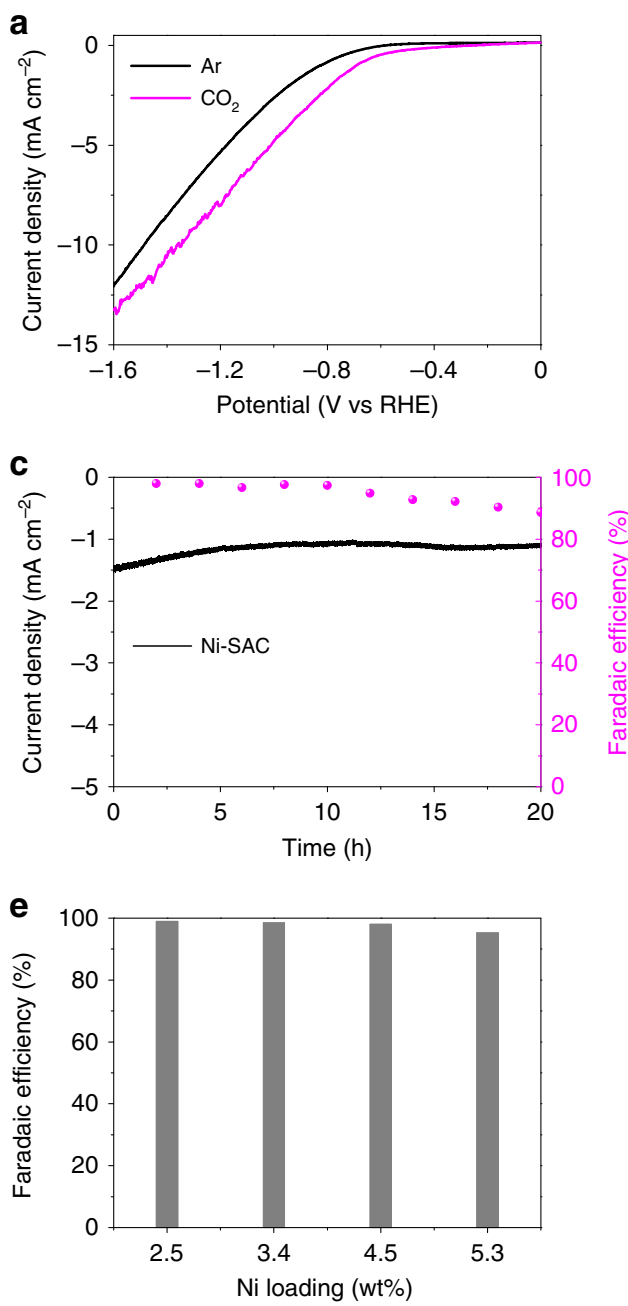
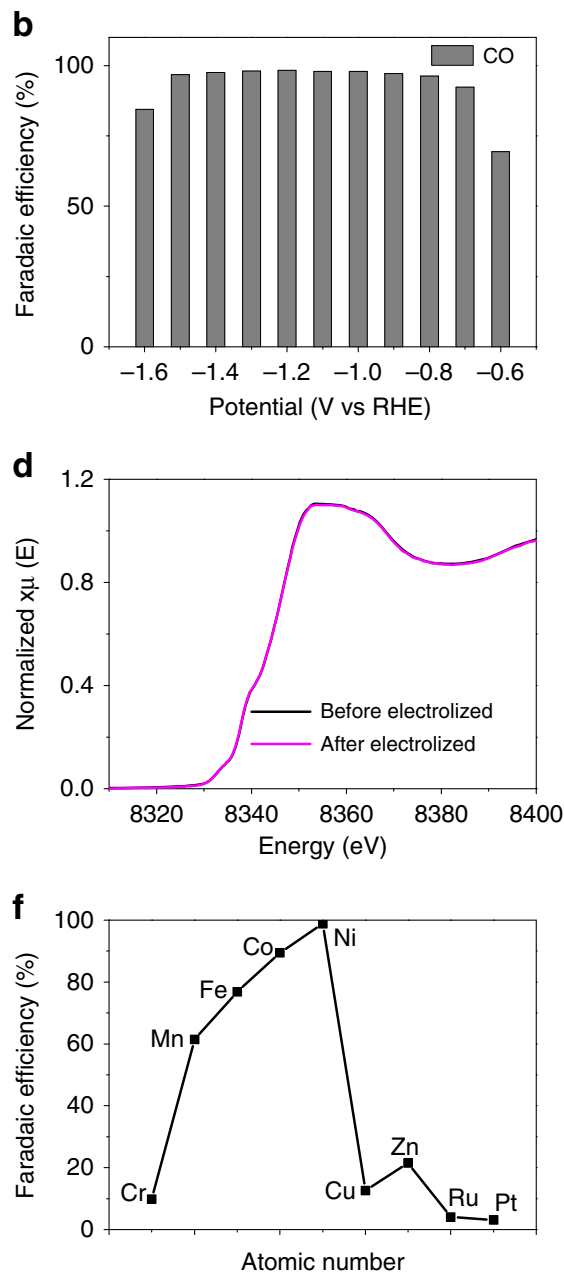

Fig. $6 \mathrm{CO}_{2}$ electrochemical reduction performance of metal single-atom catalysts. a Cathodic LSV scans for Ni-SAC-2.5 in Ar-saturated and CO aqueous $0.1 \mathrm{M} \mathrm{KHCO}_{3}$ solutions. b FE $\mathrm{CO}$ for Ni-SAC-2.5 at different potentials in a $\mathrm{CO}_{2}$-saturated aqueous $0.1 \mathrm{M} \mathrm{KHCO}$ solution. c Stability test for NiSAC- 2.5 at $-0.8 \mathrm{~V}$ vs RHE. d XANES spectra for Ni-SAC-2.5 before and after $\mathrm{CO}_{2} \mathrm{RR}$ tests. e $\mathrm{FE}_{\mathrm{CO}}$ for Ni-SAC-x at $-1.2 \mathrm{~V}$ vs RHE. $\mathbf{f} F E_{\mathrm{CO}}$ for M-SACs at $-1.2 \mathrm{~V}$ vs $\mathrm{RHE}$

confirm that all of the Ni-SAC- $x$ samples contained $\mathrm{Ni}^{2+}$ atoms fourfold coordinated by $\mathrm{N}$. In addition, since our method was based on the modification of a commercially available carbon, it is suitable for scaling up. To prove this, we performed some largescale syntheses, producing up to $1.6 \mathrm{~kg}$ of Ni-SAC in a single synthesis (Fig. 5e, with potential for further production scale-up). No metallic nickel peaks were seen in the XRD pattern of the large batch sample (Supplementary Fig. 50) or corresponding HAADF-STEM image (Fig. 5f), indicating that nickel was atomically dispersed over the carbon black.

Electroreduction of $\mathrm{CO}_{2}$ to $\mathrm{CO}$. Phthalocyanines and porphyrins, in which metal ions are surrounded fourfold by $\mathrm{N}$, are important families of enzyme-mimicking catalysts. Metal porphyrins linked by organic struts to form covalent organic frameworks are particularly active for the $\mathrm{CO}_{2}$ reduction reaction $\left(\mathrm{CO}_{2} \mathrm{RR}\right)$, a key reaction for high-density renewable energy storage and $\mathrm{CO}_{2}$ capture ${ }^{32}$. Our characterization studies above revealed that the local environment of metal atoms in the $\mathrm{M}$ SACs were very similar to that of the same metals in phthalocyanines and porphyrins. Further, here the SACs were dispersed on a carbon support with good electrical conductivity, which was expected to greatly enhance electrocatalytic performance. This motivated a detailed study of the performance of the various $M$ SACs synthesized in the current work for electrocatalytic $\mathrm{CO}_{2} \mathrm{RR}$. The electrocatalytic $\mathrm{CO}_{2} \mathrm{RR}$ activities of the different M-SACs were measured in a sealed $\mathrm{H}$-type cell, with gas chromatography used for product detection. Figure 6a shows the linear sweep voltammetry (LSV) curves for Ni-SAC in $\mathrm{Ar}$ and $\mathrm{CO}_{2}$ saturated electrolytes. The current density was much higher in the $\mathrm{CO}_{2}$ saturated electrolyte, indicating that Ni-SAC was more active for $\mathrm{CO}_{2}$ reduction than hydrogen evolution. Figure $6 \mathrm{~b}$ shows the Faradaic efficiency to $\mathrm{CO}\left(\mathrm{FE}_{\mathrm{CO}}\right)$ for Ni-SAC at different potentials. Ni-SAC displayed excellent $\mathrm{CO}_{2} \mathrm{RR}$ performance, maintaining a high $\mathrm{FE}_{\mathrm{CO}}$ (above $90 \%$ ) over a wide range of potentials (from -0.7 to $-1.5 \mathrm{~V}$ vs reversible hydrogen electrode (RHE)) and a low Faradaic efficiency to $\mathrm{H}_{2}$ (Supplementary Fig. 51), with the highest $\mathrm{FE}_{\mathrm{CO}}(98.9 \%)$ achieved at $-1.2 \mathrm{~V}$. No liquid products were detected in the aqueous phase by nuclear magnetic resonance spectroscopy (Supplementary Fig. 52), confirming a very high selectivity to $\mathrm{CO}$. The $\mathrm{FE}_{\mathrm{CO}}$ was higher than most state-of-the-art catalysts (Supplementary Table 2). We also tested the performance of Ni-SAC for the $\mathrm{CO}_{2} \mathrm{RR}$ at a fixed potential ( $-0.8 \mathrm{~V}$ vs RHE). As shown in Fig. $6 \mathrm{c}$, only a very slight decrease in the current density occurred over $20 \mathrm{~h}$. Figure $6 \mathrm{~d}$ 
shows Ni $K$-edge XANES spectra for the Ni-SAC before and after $\mathrm{CO}_{2} \mathrm{RR}$ tests. The before and after spectra were almost identical, confirming that the Ni-SAC was very stable under the conditions of $\mathrm{CO}_{2} \mathrm{RR}$.

The performance of the Ni-SACs with different Ni loadings, $\mathrm{Ni}$-SAC synthesized in large scale $(1.6 \mathrm{~kg})$, and M-SACs with different metals were also evaluated for $\mathrm{CO}_{2} \mathrm{RR}$. The $\mathrm{FE}_{\mathrm{CO}}$ for the $\mathrm{Ni}-\mathrm{SACs}$ with Ni loadings of $2.5,3.4,4.5$, and $5.3 \mathrm{wt} . \%$ at $-1.2 \mathrm{~V}$ were $98.9 \%$, 98.5\%, 98.0\%, and 95.3\%, respectively (Fig. 6e). The $\mathrm{FE}_{\mathrm{CO}}$ of the Ni-SAC-5.3 sample was a bit smaller than values determined for the other samples with lower Ni loadings, which may have been due to the co-existence of Ni-SACs and tiny $\mathrm{Ni}$ clusters in this sample. To demonstrate the scalability of the "ligand-mediated" method, we also test the $\mathrm{CO}_{2} \mathrm{RR}$ performance of the Ni-SAC synthesized in large scale $(\sim 1.6 \mathrm{~kg})$. The catalytic properties of the large batch sample were almost identical to those of samples synthesized on a 70-mg scale (Supplementary Fig. 53), confirming the scalability of the new synthetic method. Furthermore, thanks to the universality of the "ligand-mediated" method, it was possible to quantitatively compare the effect of central metal atom in M-SACs on $\mathrm{CO}_{2} \mathrm{RR}$ performance. The $\mathrm{CO}_{2} \mathrm{RR}$ performance of the M-SACs, each with similar metal loadings, were tested at $-1.2 \mathrm{~V}$ vs RHE (Fig. 6f). Interestingly, the $\mathrm{FE}_{\mathrm{CO}}$ of the M-SACs plotted against metal atomic number followed a volcano curve, with the Ni-SAC displaying the highest activity for $\mathrm{CO}_{2} \mathrm{RR}$. On transitioning from $\mathrm{Cr}$ to $\mathrm{Ni}$, the $\mathrm{FE}_{\mathrm{CO}}$ increased, whereas on going from $\mathrm{Ni}$ to $\mathrm{Zn}$ the $\mathrm{FE}_{\mathrm{CO}}$ decreased abruptly. The observation of a "volcano curve" likely originates from the d-band center position of each metal in the M-SACs, with the d-band center position of $\mathrm{Ni}^{2+}$ in the Ni-SACs being most ideal for $\mathrm{CO}_{2} \mathrm{RR}^{33,34}$.

\section{Discussion}

As mentioned above, a wide range of $\mathrm{M}$-SACs $(\mathrm{M}=\mathrm{Ni}, \mathrm{Mn}, \mathrm{Fe}$, $\mathrm{Co}, \mathrm{Cr}, \mathrm{Cu}, \mathrm{Zn}, \mathrm{Ru}, \mathrm{Pt}$, and combinations thereof) were successfully prepared using a single "ligand-mediated" synthetic strategy. In this strategy, metal complexes (i.e., $\mathrm{M}^{2+}$ ions coordinated by 1,10-phenanthroline ligands) facilitate the creation of "porphyrin-like" single metal atom sites on the carbon black surface, thereby tightly binding the metal cations and preventing the aggregation of the metal atoms into clusters or nanoparticles. Further, the direct use of a conductive carbon support circumvents the need to use high pyrolysis temperatures that are obviously detrimental to SACs' stability (typically temperatures as high as $900^{\circ} \mathrm{C}$ are needed to produce carbon materials with good conductivities if derived from ZIF precursors). HAADF-STEM and EXAFS analysis indicated that the metal atoms were atomically dispersed (as $\mathrm{M}^{2+}$ ) in all the M-SACs. The developed synthetic method thus confers the following advantages over existing methods for SAC fabrication: (1) it is universal and can be applied to synthesize various transition metal SACs and even bimetallic SACs; (2) commercially available, conductive carbons can be used as a support, which is advantageous for electrocatalytic applications; (3) the method allows large-scale SAC production (kilogram scale, with potential for further production scale-up); and (4) the method yields SACs with high metal loadings. Therefore, this work represents a totally new paradigm for the synthesis of SACs.

In summary, we have developed a universal and robust "ligand-mediated" method for the synthesis of M-SACs with high metal contents. The method can be used to synthesize M-SACs containing first-, second- and third-row transition metals on carbon supports. Ni-SACs synthesized by the new method show excellent activity and stability for electrochemical $\mathrm{CO}_{2}$ reduction to $\mathrm{CO}\left(\mathrm{FE}_{\mathrm{CO}}\right.$ of $98.9 \%$ at $-1.2 \mathrm{~V}$ at the optimum Ni loading of 2.5 wt.\%). As well as M-SACs containing single metals (Cr, Mn, Fe, $\mathrm{Co}, \mathrm{Cu}, \mathrm{Zn}, \mathrm{Ru}, \mathrm{Pt}$ ), the synthetic method could also be used to synthesize bimetallic SACs. Results pave the way for the largescale fabrication of M-SACs with high metal loadings for $\mathrm{CO}_{2}$ reduction, oxygen reduction or evolution, hydrogen evolution, $\mathrm{N}_{2}$ reduction, and other chemical reactions.

\section{Methods}

Chemicals. Nickel (II) acetate tetrahydrate and manganese (II) acetate tetrahydrate were purchased from Shanghai Macklin Biochemical Technology Co., Ltd. Iron (II) acetate was purchased from J\&K Scientific., Ltd. Cobalt (II) acetate tetrahydrate was purchased from Guangdong GHTECH Co., Ltd. Chromium (III) nitrate nonahydrate and copper (II) acetate monohydrate were purchased from Xilong Scientific Co., Ltd. Ruthenium (III) chloride was purchased from Innochem Co., Ltd. Platinum (II) chloride was purchased from Acros Organic Co., Ltd. 1,10Phenanthroline monohydrate was purchased from Sinopharm Chemical Reagent Co., Ltd. Zinc (II) acetate dihydrate, ethanol, and dimethyl sulfoxide were purchased from Beijing Chemical Works. All reagents and solvents were of analytical grade and used as received without additional purification. The $\mathrm{CO}_{2}$ and $\mathrm{Ar}$ feed gases were purchased from Beijing SIDADE RM Science and Technology Co., Ltd.

Synthesis of Ni-SAC-x (where $\boldsymbol{x}$ is the wt.\% Ni). For the synthesis of Ni-SAC $2.5 \%$ (i.e., a Ni SAC containing 2.5 wt.\% Ni), nickel (II) acetate tetrahydrate (12.4 $\mathrm{mg})$ and 1,10-phenanthroline monohydrate $(29.7 \mathrm{mg})$ were dissolved in $2 \mathrm{~mL}$ of ethanol followed by stirring for approximately $20 \mathrm{~min}$ at room temperature. Subsequently, carbon black $(69.6 \mathrm{mg})$ was added into the solution, and the resulting solution was heated in an oil-bath at $60^{\circ} \mathrm{C}$ for $4 \mathrm{~h}$ under continuous magnetic stirring. The resulting dispersion was then heated at $80^{\circ} \mathrm{C}$ in air for $12 \mathrm{~h}$ to evaporate the ethanol, yielding a black solid. The black solid obtained was lightly ground with a mortar and pestle, then transferred into a ceramic crucible and placed in a tube furnace. The black solid was then heated to $600{ }^{\circ} \mathrm{C}$ at a rate of $10^{\circ}$ $\mathrm{C} \min ^{-1}$ under an argon atmosphere and then held at $600{ }^{\circ} \mathrm{C}$ for $2 \mathrm{~h}$. The product obtained after cooling to room temperature was denoted as Ni-SAC-2.5\%. The NiSAC-3.4\%, Ni-SAC-4.5\%, and Ni-SAC-5.3\% samples were obtained using a similar procedure, except that the amount of nickel (II) acetate tetrahydrate used was increased to $18.7,24.9$, and $49.7 \mathrm{mg}$, respectively, and the amount of 1,10 -phenanthroline monohydrate used was increased proportionally to $44.6,59.5$, and $118.9 \mathrm{mg}$, respectively, to maintain a 1,10-phenanthroline:Ni molar ratio of 3 .

Synthesis of Mn-SAC, Fe-SAC, Co-SAC, and Zn-SAC. The Mn-SAC, Fe-SAC, $\mathrm{Co}-\mathrm{SAC}$, and $\mathrm{Zn}$-SAC samples were prepared using a similar procedure to that described above for Ni-SAC-2.5, except that the amount of 1,10-phenanthroline monohydrate and metal salt were adjusted as required to achieve a 1,10-phenanthroline:M molar ratio of 3 . The amounts of metal salt and 1,10-phenanthroline monohydrate used in each synthesis were: manganese (II) acetate tetrahydrate (19.8 $\mathrm{mg})$ and 1,10-phenanthroline monohydrate $(101.9 \mathrm{mg})$; iron (II) acetate (13.8 mg) and 1,10-phenanthroline monohydrate (100.3 mg); cobalt (II) acetate tetrahydrate $(18.8 \mathrm{mg})$ and 1,10-phenanthroline monohydrate $(94.99 \mathrm{mg})$; and zinc (II) acetate dihydrate $(14.9 \mathrm{mg})$ and 1,10-phenanthroline monohydrate $(85.6 \mathrm{mg}$ )

Synthesis of Cr-SAC, Cu-SAC, Ru-SAC, and Pt-SAC. Cr-SAC, Cu-SAC, Ru-SAC and Pt-SAC were prepared using a similar procedure to that described for Ni-SAC2.5 , except that dimethyl sulfoxide rather than ethanol was used as the solvent. The amounts of metal salt and 1,10-phenanthroline monohydrate used in each synthesis were: chromium (III) nitrate nonahydrate $(34.2 \mathrm{mg})$ and 1,10 -phenanthroline monohydrate $(107.7 \mathrm{mg})$; copper (II) acetate monohydrate $(13.9 \mathrm{mg})$ and 1,10-phenanthroline monohydrate ( $88.1 \mathrm{mg})$; ruthenium (III) chloride (9.1 $\mathrm{mg}$ ) and 1,10-phenanthroline monohydrate $(55.4 \mathrm{mg})$; and platinum (II) chloride $(6.1 \mathrm{mg})$ and 1,10-phenanthroline monohydrate $(28.7 \mathrm{mg})$. Owing to the higher boiling point of dimethyl sulfoxide, the solvent evaporation step (that preceded sample heating in the tube furnace) was performed at $190^{\circ} \mathrm{C}$ instead of $80^{\circ} \mathrm{C}$.

Synthesis of bimetallic SACs. Fe/Co-SAC was prepared using a similar procedure to that described for $\mathrm{Ni}$-SAC-2.5, except that the amount of metal salt was adjusted to iron (II) acetate (13.8 mg) and cobalt (II) acetate tetrahydrate $(18.8 \mathrm{mg})$, and the amount of 1,10-phenanthroline monohydrate was adjusted to $195.29 \mathrm{mg}$. $\mathrm{Ru} / \mathrm{Fe}-$ $\mathrm{SAC}, \mathrm{Ru} / \mathrm{Co}-\mathrm{SAC}$, and $\mathrm{Ru} / \mathrm{Ni}-\mathrm{SAC}$ were prepared using a similar procedure to that described for Ni-SAC-2.5, except that the dimethyl sulfoxide rather than ethanol was used as the solvent. The amounts of metal salt and 1,10-phenanthroline monohydrate used in each synthesis were: ruthenium (III) chloride $(9.1 \mathrm{mg})$, iron (II) acetate $(13.8 \mathrm{mg})$, and 1,10-phenanthroline monohydrate $(155.7 \mathrm{mg})$; ruthenium (III) chloride $(9.1 \mathrm{mg})$, cobalt (II) acetate tetrahydrate $(18.8 \mathrm{mg})$, and 1,10 phenanthroline monohydrate $(150.39 \mathrm{mg})$; and ruthenium (III) chloride $(9.1 \mathrm{mg})$, nickel (II) acetate tetrahydrate $(12.4 \mathrm{mg})$, and 1,10-phenanthroline monohydrate $(85.1 \mathrm{mg})$. 
Synthesis of Ni-SAC on a large scale. For the synthesis of Ni-SAC on a large scale, nickel (II) acetate tetrahydrate (165.0 g) and 1,10-phenanthroline monohydrate $(920.7 \mathrm{~g})$ were dissolved in $2.5 \mathrm{~L}$ of ethanol followed by stirring at room temperature. Subsequently, carbon black $(924.0 \mathrm{~g})$ was added into the solution, and the resulting solution was then heated at $60^{\circ} \mathrm{C}$ for $4 \mathrm{~h}$ under continuous stirring. The resulting dispersion was then heated at $80^{\circ} \mathrm{C}$ in air for $12 \mathrm{~h}$ to evaporate ethanol, yielding a black solid that was ground to a powder. The black powder was then heated to $600^{\circ} \mathrm{C}$ at a rate of $10^{\circ} \mathrm{C} \mathrm{min}-1$ under an argon atmosphere, and then held at $600^{\circ} \mathrm{C}$ for $2 \mathrm{~h}$. The product was obtained after cooling to room temperature.

Catalyst characterization. TEM images were collected on a HITACHI HT-7700 (HITACHI, Japan) microscope operating at an accelerating voltage of $100 \mathrm{kV}$. HAADF-STEM images and EDX elemental maps were obtained on an ARM200CF (JEOL, Tokyo, Japan) operating at $200 \mathrm{kV}$ and equipped with double spherical aberration correctors. The resolution of the probe defined by the objective pre-field was $78 \mathrm{pm}$. XRD patterns were obtained on a Bruker D8 Focus X-ray diffractometer equipped with $\mathrm{Cu}$ Ka radiation source $(\lambda=1.5405 \AA)$ operating at $40 \mathrm{kV}$. XPS data were collected on a VGESCALABMKII X-ray photoelectron spectrometer using a non-monochromatized Al-Ka X-ray source $(\mathrm{h} v=1486.6 \mathrm{eV})$. XAFS data were obtained at the Beijing Synchrotron Radiation Facility (1W1B). Raman spectra were collected on a Renishaw inVia-Reflex spectrometer system and excited by a $532-\mathrm{nm}$ laser. The metal loadings in the SACs were measured by ICPOES (Varian 710).

Electrochemical measurements. A three-electrode sealed $\mathrm{H}$-type cell was used in all electrochemical tests. Catalysts $(4 \mathrm{mg})$ were dispersed in a solution of $\mathrm{H}_{2} \mathrm{O}$ $(0.5 \mathrm{~mL})$, ethanol $(0.48 \mathrm{~mL})$, and $5 \mathrm{wt} . \%$ Nafion solution $(0.02 \mathrm{~mL})$. After sonication for $2 \mathrm{~h}$, a uniform ink-like dispersion was obtained. Next, $2 \mu \mathrm{L}$ of the dispersion was dropped onto a polished glassy carbon electrode (GCE), and the resulting electrode was allowed to dry in air for several hours. This catalyst/GCE served as the working electrode in subsequent electrochemical tests. $\mathrm{An} \mathrm{Ag} / \mathrm{AgCl}$ electrode and a Pt wire were used as the reference electrode and the counter electrode, respectively. LSV data were collected at a scan rate of $10 \mathrm{mV} \mathrm{s}^{-1}$ in a 0.1 $\mathrm{M} \mathrm{KHCO}_{3}$ electrolyte. When testing the Faradaic efficiency, the working electrode was held at a constant potential for $30 \mathrm{~min}$. The gas products evolved were detected by a Shimadzu GC-2014 chromatograph (Shimadzu Co., Japan) equipped with a $\mathrm{HP}$ PLOT $\mathrm{Al}_{2} \mathrm{O}_{3}$ column and a flame ionization detector.

\section{Data availability}

All relevant data are available from the corresponding author on request.

Received: 2 June 2019; Accepted: 13 September 2019;

Published online: 08 October 2019

\section{References}

1. Wang, A., Li, J. \& Zhang, T. Heterogeneous single-atom catalysis. Nat. Rev. Chem. 2, 65-81 (2018).

2. Zhu, C., Fu, S., Shi, Q., Du, D. \& Lin, Y. Single-atom electrocatalysts. Angew. Chem. Int. Ed. 56, 13944-13960 (2017).

3. Chen, Y. et al. Single-atom catalysts: synthetic strategies and electrochemical applications. Joule 2, 1242-1264 (2018).

4. Gao, D. et al. Size-dependent electrocatalytic reduction of $\mathrm{CO}_{2}$ over Pd nanoparticles. J. Am. Chem. Soc. 137, 4288-4291 (2015).

5. Huang, X. et al. Freestanding palladium nanosheets with plasmonic and catalytic properties. Nat. Nanotechnol. 6, 28-32 (2011).

6. Chen, F., Jiang, X., Zhang, L., Lang, R. \& Qiao, B. Single-atom catalysis: bridging the homo- and heterogeneous catalysis. Chin. J. Catal. 39, 893-898 (2018).

7. Liu, L. \& Corma, A. Metal catalysts for heterogeneous catalysis: from single atoms to nanoclusters and nanoparticles. Chem. Rev. 118, 4981-5079 (2018).

8. Qiao, B. et al. Single-atom catalysis of $\mathrm{CO}$ oxidation using $\mathrm{Pt}_{1} / \mathrm{FeO}_{\mathrm{x}}$. Nat. Chem. 3, 634-641 (2011)

9. Wei, S. et al. Direct observation of noble metal nanoparticles transforming to thermally stable single atoms. Nat. Nanotechnol. 13, 856-861 (2018).

10. Wan, J. et al. Defect effects on $\mathrm{TiO}_{2}$ nanosheets: stabilizing single atomic site $\mathrm{Au}$ and promoting catalytic properties. Adv. Mater. 30, 1705369 (2018).

11. Xie P. et al. Nanoceria-supported single-atom platinum catalysts for direct methane conversion. ACS Catal. 8, 4044-4048 (2018).

12. Zhao, C. et al. Ionic exchange of metal-organic frameworks to access single nickel sites for efficient electroreduction of $\mathrm{CO}_{2}$. J. Am. Chem. Soc. 139, 8078-8081 (2017).

13. Li, X. et al. Exclusive Ni- $\mathrm{N}_{4}$ sites realize near-unity $\mathrm{CO}$ selectivity for electrochemical $\mathrm{CO}_{2}$ reduction. J. Am. Chem. Soc. 139, 14889-14892 (2017).
14. Yang, H. B. et al. Atomically dispersed $\mathrm{Ni}(\mathrm{i})$ as the active site for electrochemical $\mathrm{CO}_{2}$ reduction. Nat. Energy 3, 140-147 (2018).

15. Jiang, $\mathrm{K}$. et al. Isolated $\mathrm{Ni}$ single atoms in graphene nanosheets for highperformance $\mathrm{CO}_{2}$ reduction. Energy Environ. Sci. 11, 893-903 (2018).

16. Gao, G., Jiao, Y., Waclawik, E. R. \& Du, A. Single atom (Pd/Pt) supported on graphitic carbon nitride as an efficient photocatalyst for visible-light reduction of carbon dioxide. J. Am. Chem. Soc. 138, 6292-6297 (2016).

17. Chen, W. et al. Rational design of single molybdenum atoms anchored on Ndoped carbon for effective hydrogen evolution reaction. Angew. Chem. Int. Ed. 56, 16086-16090 (2017)

18. Yin, P. et al. Single cobalt atoms with precise $\mathrm{N}$-coordination as superior oxygen reduction reaction catalysts. Angew. Chem. Int. Ed. 55, 10800-10805 (2016).

19. Geng, Z. et al. Achieving a record-high yield rate of 120.9 ug NH3 mgcat.- ${ }^{-1} \mathrm{~h}^{-1}$ for $\mathrm{N}_{2}$ electrochemical reduction over Ru single-atom catalysts. Adv. Mater. 30, 1803498 (2018).

20. Stefan, V. \& White, M. G. Catalysis applications of size-selected cluster deposition. ACS Catal. 5, 7152-7176 (2015).

21. Sun, S. H. et al. Single-atom catalysis using Pt/graphene achieved through atomic layer deposition. Sci. Rep. 3, 1775 (2013).

22. Zhu, Y. et al. A cocoon silk chemistry strategy to ultrathin N-doped carbon nanosheet with metal single-site catalysts. Nat. Commun. 9, 3861 (2018).

23. Shang, L. et al. Well-dispersed ZIF-derived Co,N-Co-doped carbon nanoframes through mesoporous-silica-protected calcination as efficient oxygen reduction electrocatalysts. Adv. Mater. 28, 1668-1674 (2016).

24. Chen, $\mathrm{Y}$. et al. Isolated single iron atoms anchored on $\mathrm{N}$-doped porous carbon as an efficient electrocatalyst for the oxygen reduction reaction. Angew. Chem. Int. Ed. 56, 6937-6941 (2017).

25. Liu, W. et al. Single-atom dispersed Co-N-C catalyst: structure identification and performance for hydrogenative coupling of nitroarenes. Chem. Sci. 7 , 5758-5764 (2016)

26. Fei, $\mathrm{H}$. et al. General synthesis and definitive structural identification of $\mathrm{MN}_{4} \mathrm{C}_{4}$ single-atom catalysts with tunable electrocatalytic activities. Nat. Catal. 1, 63-72 (2018).

27. Qu, Y. et al. Direct transformation of bulk copper into copper single sites via emitting and trapping of atoms. Nat. Catal. 1, 781-786 (2018).

28. Conghui, T. et al. A stable nanocobalt catalyst with highly dispersed $\mathrm{CoN}_{\mathrm{x}}$ active sites for the selective dehydrogenation of formic acid. Angew. Chem. Int. Ed. 56, 16616-16620 (2017).

29. Li, Z. et al. Co-based catalysts derived from layered-double-hydroxide nanosheets for the photothermal production of light olefins. Adv. Mater. 30, e1800527 (2018).

30. Deng, Y., Handoko, A. D., Du, Y., Xi, S. \& Yeo, B. S. In situ raman spectroscopy of copper and copper oxide surfaces during electrochemical oxygen evolution reaction: identification of $\mathrm{Cu}^{\mathrm{III}}$ oxides as catalytically active species. ACS Catal. 6, 2473-2481 (2016).

31. Sasaki, K., Marinkovic, N., Isaacs, H. S. \& Adzic, R. R. Synchrotron-based in situ characterization of carbon-supported platinum and platinum monolayer electrocatalysts. ACS Catal. 6, 69-76 (2015).

32. Lin, S. et al. Covalent organic frameworks comprising cobalt porphyrins for catalytic $\mathrm{CO}_{2}$ reduction in water. Science 349, 1208-1213 (2015).

33. Zheng, Y. et al. Molecule-level g- $\mathrm{C}_{3} \mathrm{~N}_{4}$ coordinated transition metals as a new class of electrocatalysts for oxygen electrode reactions. J. Am. Chem. Soc. 139, 3336-3339 (2017).

34. Hammer, B. \& Norskov, J. K. Theoretical surface science and catalysiscalculations and concepts. Adv. Catal. 45, 71-129 (2000).

\section{Acknowledgements}

The authors are grateful for financial support from the National Key Projects for Fundamental Research and Development of China (2018YFB1502002, 2017YFA0206904, 2017YFA0206900, 2016YFB0600901), the National Natural Science Foundation of China (51825205, 51772305, 51572270, U1662118, 21871279, 21802154), the Beijing Natural Science Foundation $(2191002,2182078,2194089)$, the Strategic Priority Research Program of the Chinese Academy of Sciences (XDB17000000), the Royal Society-Newton Advanced Fellowship (NA170422), the International Partnership Program of Chinese Academy of Sciences (GJHZ1819, GJHZ201974), the Beijing Municipal Science and Technology Project (Z181100005118007), the K. C. Wong Education Foundation, and the Youth Innovation Promotion Association of the CAS. The XAFS experiments were conducted in 1W1B beamline of Beijing Synchrotron Radiation Facility (BSRF). G.I.N.W. acknowledges funding support from Greg and Kathryn Trounson (via a generous philanthropic donation), the Energy Education Trust of New Zealand, and the MacDiarmid Institute for Advanced Materials and Nanotechnology.

\section{Author contributions}

T.Z. and L.S. conceived the idea and designed the experiments. T.Z. supervised the project. H.Y. and L.S. carried out the experiments and characterizations. Q.Z. and L.G. 
helped to carry out the spherical aberration-corrected HAADF-STEM characterizations. H.Y., L.S., G.I.N.W. and T.Z. wrote the manuscript. All the authors discussed the results and commented on the manuscript.

\section{Competing interests}

The authors declare no competing interests.

\section{Additional information}

Supplementary information is available for this paper at https://doi.org/10.1038/s41467019-12510-0.

Correspondence and requests for materials should be addressed to T.Z.

Peer review information Nature Communications thanks anonymous reviewers for their contributions to the peer review of this work.

Reprints and permission information is available at http://www.nature.com/reprints
Publisher's note Springer Nature remains neutral with regard to jurisdictional claims in published maps and institutional affiliations.

(c) (i) Open Access This article is licensed under a Creative Commons Attribution 4.0 International License, which permits use, sharing, adaptation, distribution and reproduction in any medium or format, as long as you give appropriate credit to the original author(s) and the source, provide a link to the Creative Commons license, and indicate if changes were made. The images or other third party material in this article are included in the article's Creative Commons license, unless indicated otherwise in a credit line to the material. If material is not included in the article's Creative Commons license and your intended use is not permitted by statutory regulation or exceeds the permitted use, you will need to obtain permission directly from the copyright holder. To view a copy of this license, visit http://creativecommons.org/ licenses/by/4.0/.

(C) The Author(s) 2019 